\title{
Glycogen granules in resting and inflammatory rainbow trout phagocytes - an ultrastructural study
}

\author{
António Afonso ${ }^{1,2, *}$, Paula M. Macedo ${ }^{1}$, Anthony E. Ellis ${ }^{3}$, Manuel T. Silva ${ }^{1}$ \\ ${ }^{1}$ Institute for Molecular and Cell Biology, University of Porto, Rua do Campo Alegre 823, 4150-180 Porto, Portugal \\ ${ }^{2}$ Abel Salazar Biomedical Institute, University of Porto, Largo Abel Salazar 2, 4099-003 Porto, Portugal \\ ${ }^{3}$ Marine Laboratory, Fisheries Research Services, PO Box 101, Victoria Rd, Aberdeen AB11 9DB, United Kingdom
}

\begin{abstract}
The ultrastructural image of glycogen granules in the cytoplasm of rainbow trout phagocytes in sections stained by the conventional lead or uranyl-lead stains is highly dependent on fixation conditions, the granules being visible only when adequate fixation protocols are used. Morphometry of samples processed for the detection of peroxidase or esterase activities (to specifically label neutrophils and macrophages, respectively), and simultaneously stained for the specific detection of glycogen, showed that inflammatory peritoneal neutrophils were richer in glycogen granules than resting neutrophils. This increase in glycogen content occurs after the migration from the haematopoietic tissues and peripheral blood to the inflamed foci. Glycogen granules could not be found in resting peritoneal macrophages but were found in inflammatory macrophages. The macrophage granules occurred in smaller amounts than in neutrophils, and consisted of granules identical to those of neutrophils together with significantly smaller granules. No evidence for the utilization of glycogen by neutrophils phagocytosing bacteria within the peritoneal cavity was found.
\end{abstract}

KEY WORDS: Glycogen granules $\cdot$ Neutrophils $\cdot$ Macrophages $\cdot$ Phagocytosis $\cdot$ Fish

Resale or republication not permitted without written consent of the publisher

\section{INTRODUCTION}

Farmed fish are an important source of animal protein for human consumption, but the intensive conditions on fish farms can be conducive to infections with severe economic consequences. Phagocytosis, killing and degradation of pathogenic microorganisms is a fundamental mechanism of host defense against infection in animals. Two distinct phagocytic cell populations are present in vertebrates, the macrophage and the neutrophil. Glycogen is used as a store of glucose in several cells, and, in the case of neutrophils and macrophages, glycogen-derived glucose can be utilized for the respiratory burst that accompanies phagocytosis (Scott 1968, Wilkinson 1977, Weisdorf et al. 1982).

It has been reported that inflammatory neutrophils of mammals (Scott \& Cooper 1974, Robinson et al. 1982) and the eel (Nagamura \& Wakabayashi 1985, Hine \& Wain 1988) contain more glycogen than resting neu-

*E-mail: aafonso@ibmc.up.pt trophils. This increase in the storage molecule would be useful for the metabolic activities of the neutrophils phagocytosing under the anaerobic conditions which frequently are faced by the phagocytes (Suzuki \& Iida 1992).

A few reports deal with the presence of glycogen in fish phagocytes, but most are light microscopic studies (as reviewed by Ellis 1977, MacArthur \& Fletcher 1985, Rowley et al. 1988). Extending our previous results regarding the cytochemical characterization of resting and inflammatory rainbow trout phagocytes, here we analyze by ultrastructural methods the glycogen pools of neutrophils and macrophages in the blood and peritoneal cavity.

\section{MATERIALS AND METHODS}

Fish. Sexually immature rainbow trout Oncorhynchus mykiss, weighing between 100 and $200 \mathrm{~g}$, were purchased from a commercial farm. The fish were 
maintained in recirculating aerated fresh water, at $15^{\circ} \mathrm{C}$, in $7000 \mathrm{l}$ fibreglass tanks. Water quality was maintained with mechanical and biological filtration and fish were fed ad libitum on commercial pellets. The fish to be injected with phlogistic agents were transferred to $600 \mathrm{l}$ fibreglass tanks under the same conditions as above, where they were maintained for at least $15 \mathrm{~d}$ before the study. Only healthy fish, as indicated by their activity and external appearance, were used.

Phagocytes. The present results concern phagocytes under resting or transient inflammatory situations. Resting neutrophils and monocytes/macrophages were studied in the leucocyte populations of the blood and the resting peritoneal cavities of normal, noninjected, rainbow trout. Inflammatory neutrophils and macrophages were collected from peritoneal cavities rendered inflamed by the intraperitoneal (i.p.) injection of bacterial or non-bacterial phlogistic agents. The peritoneal phagocyte responses following the i.p. injection of the agents used in this study were previously described in detail (Afonso et al. 1998a).

Intraperitoneal injection of phlogistic agents. Undiluted Incomplete Freund's Adjuvant (IFA) and suspensions of live or formol-killed Yersinia ruckeri strain FP-3 type $1\left(5 \times 10^{7}\right.$ bacteria fish $\left.^{-1}\right)$ were injected i.p. in volumes of $100 \mu \mathrm{l}$. The bacterial strain was kindly supplied by Prof. J. Barja (University of Santiago de Compostela, Spain). Under the conditions described, and as previously reported (Afonso et al. 1998a), the i.p. injection of $Y$. ruckeri does not induce a progressive infection. Suspensions of live or formolkilled bacteria to be injected into the peritoneal cavities of trout were prepared as previously described (Afonso et al. 1998a,b). In some experiments, $24 \mathrm{~h}$ after the i.p. injection of killed $5 \times 10^{7} Y$. ruckeri, fish were injected i.p. with $4 \times 10^{8}$ live Y. ruckeri to promote the phagocytosis of bacteria by neutrophils (Afonso et al. 1998a).

All agents were injected as described (Afonso et al. 1998a), into the peritoneal cavity of fish under anaesthesia with $0.3 \mathrm{ml} \mathrm{l}^{-1}$ of ethylene glycol monophenyl ether (Merck). Immediately after the i.p. injection the fish were returned to their tank.

Collection of peritoneal leucocytes. The peritoneal cells were collected from untreated trout and at intervals (see 'Results') after the i.p. injections by a previously described procedure (Afonso et al. 1997, 1998a). The peritoneal cell suspensions were analysed within minutes after collection for morphological and cytochemical parameters as described below.

Study of blood leucocytes. Blood was collected from the caudal vein of anaesthetised trout using heparinized syringes. Leucocytes in the blood of normal rainbow trout or of trout injected i.p. with the bacterial inocula were obtained by the method of Thuvander et al. (1987), and these leucocyte preparations were analysed for peroxidase, esterase and glycogen by the methods described below.

Electron microscopy of leucocytes. Blood or peritoneal leucocyte suspensions in phosphate buffered saline (PBS) were centrifuged at $1000 \times g$ for $10 \mathrm{~min}$ and the pellet was fixed by the following methods: (1) Routinely, samples were fixed by our standard procedure as previously described (Silva \& Macedo 1983). This involves prefixation with $4 \%$ paraformaldehyde$1.25 \%$ glutaraldehyde- $10 \mathrm{mM}$ calcium chloride in $50 \mathrm{mM}$ cacodylate buffer for $4 \mathrm{~h}$ at $4{ }^{\circ} \mathrm{C}$; fixation at room temperature for $2 \mathrm{~h}$ with $1 \% \mathrm{OsO}_{4}$ in veronal-acetate buffer ( $\mathrm{pH}$ 6.4) supplemented with $10 \mathrm{mM}$ calcium chloride; and postfixation, at room temperature, with $1 \%$ aqueous uranyl acetate for $1 \mathrm{~h}$ (Silva et al. 1971). Between prefixation and fixation, the samples were washed twice with $50 \mathrm{mM}$ cacodylate buffer supple-

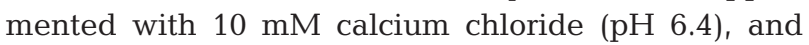
between fixation and postfixation they were washed once with water. To analyse the influence of fixation methods on the ultrastructural image of glycogen granules, several other fixation protocols were used as follows: (2) As in (1) but omitting the uranyl postfixation. (3) As in (1) but prefixation with $0.25 \%$ glutaraldehyde; this protocol was also used for the cytochemical technique for peroxidase (see below). (4) As in (1) but prefixation with $1.5 \%$ glutaraldehyde. (5) Fixation only with $1 \%$ osmium tetroxide as in (1). (6) As in (1) but osmium tetroxide fixation carried out for $2 \mathrm{~h}$ at room temperature with $2 \% \mathrm{OsO}_{4}$ in $0.1 \mathrm{M}$ cacodylate buffer $\mathrm{pH} 7.3$, supplemented with $3 \%$ potassium ferrocyanide (Karnowsky 1971, Robinson et al. 1982). In protocols (3) to (5) fixation was or was not followed by a postfixation at room temperature with $1 \%$ aqueous uranyl acetate for $1 \mathrm{~h}$ (Silva et al. 1971).

After dehydration in ethanol, the samples were embedded in Epon and ultrathin sections were cut with an LKB Ultratome III microtome. The sections were contrasted with uranyl acetate followed by lead citrate (Silva et al. 1987) or with lead citrate alone, as indicated in the legends to the figures. Observations and micrographs were done with a Zeiss EM 10C electron microscope operating at $60 \mathrm{kV}$.

Cytochemistry. Ultrastructural detection of peroxidase activity was carried out by the method of Robbins et al. (1971), with an initial fixation in 0.25 or $1.5 \%$ glutaraldehyde for $15 \mathrm{~min}$ at $4^{\circ} \mathrm{C}$.

Ultrastructural detection of alpha-naphthyl butyrate (ANB) esterase was carried out as described by Bozdech \& Bainton (1981), with fixation with $1 \%$ osmium tetroxide in distilled water with $\mathrm{pH}$ adjusted to 7.6 to heighten the contrast of the reaction product (Bozdech \& Bainton 1981). 
The ultrastructural staining of cytoplasmic polysaccharides was carried out using the original method of Thiéry (1967), or a modification (Silva \& Macedo 1987). These techniques, which specifically label, at the electron microscope level, periodic acid Schiff (PAS)-positive molecules (Thiéry 1967), were applied to sections of samples fixed by the methods described above for conventional electron microscopy or processed for peroxidase or ANB esterase ultrastructural cytochemistry. For the Thiéry staining, the sections were mounted on gold grids and were sequentially treated with: $1 \%$ aqueous periodic acid for $30 \mathrm{~min}$, $0.2 \%$ thiocarbohydrazide in $20 \%$ acetic acid for $2 \mathrm{~h}$, and $1 \%$ aqueous silver proteinate (Thiéry 1967) or $1 \%$ silver vitelinate (Silva \& Macedo 1987) for $30 \mathrm{~min}$ in the dark.

Morphometry. To quantify the amount of glycogen in neutrophils and macrophages, the morphometric method described by Robinson et al. (1982) was used. Electron micrograph prints of Thiéry-stained sections of samples processed for the peroxidase detection were made at a magnification of 20000 . The number of granules in cytoplasmic portions of median sections of the phagocytes with a known area was scored and converted to number of granules $\mu \mathrm{m}^{-2}$.

Glucose assay in plasma and peritoneal fluid. Glucose concentrations were determined by the hexokinase method using a diagnostic kit (Sigma, Madrid, Spain), in supernatants of exudates of inflamed peritoneal cavities and in plasma from heparinized venous blood of the same fish $(n=5)$, collected as described above. The determinations were carried out in trout that were injected i.p. with 2 consecutive inocula of Yersinia ruckeri, as described above.

Statistics. Values were analysed by the Student's $t$-test.

\section{RESULTS}

Glycogen granules observed in the cytoplasmic matrix of neutrophils and macrophages were of the $\beta$ type (Drochmans 1962) (Figs. 1, 3A, 4C \& 5). The diameter of neutrophil glycogen granules in ultrathin sections of samples prepared according to protocol (1) and stained by the Thiéry's technique was $35.72 \pm$ $3.20 \mathrm{~nm}(\mathrm{n}=36)$. Inflammatory macrophages in these samples contained granules with the same size as those of neutrophils as well as smaller granules $(25.53 \pm 3.81 \mathrm{~nm} ; \mathrm{n}=14 ; \mathrm{p}<0.001)$ (Fig. 4C). Occasionally, clusters of polysaccharide granules were found in phagocytic vacuoles within macrophages or in intramacrophagic neutrophils, as reported elsewhere (Afonso et al. 1998b); these granules were of the larger type.

\section{Influence of sample processing methods on the ultrastructure of glycogen granules}

For sections contrasted by the conventional lead or uranyl-lead stains, the character of polysaccharide staining was dependent on the fixation method used to prepare the samples, the staining ranging from electron-dense (granules denser than the surrounding cytoplasmic matrix; Figs. 1 \& 3A) to electron-translucent (electron-density below the surrounding cytoplasm; Figs. 2, 3C \& 4A,B).

In samples processed by methods (1) and (5), glycogen appeared as electron-translucent areas (Fig. 4B). This aspect was also seen in sections of samples processed for the detection of peroxidase activity using fixation with $0.25 \%$ glutaraldehyde (Figs. 3C \& 4A), and whenever a postfixation with uranyl acetate was used (for example, Fig. 4B). When a prefixation with 1.5\% glutaraldehyde was followed by osmium tetroxide fixation and with exclusion of uranyl acetate postfixation, a rather high electron density was seen (Fig. 3B). The highest electron density of the granules was found when the osmium tetroxide fixative was supplemented with potassium ferrocyanide (Figs. 1 \& 3A).

Thiéry staining clearly showed the presence of PASpositive material in samples prepared with the fixation protocols described above that resulted in electrontransparent images of polysaccharide granules (Fig. 5).

\section{Differences in glycogen amount and distribution in resting and inflammatory phagocytes}

As previously reported (Afonso et al. 1998a), the distinction between neutrophils and macrophages, in peritoneal cavities of rainbow trout, particularly in inflamed cavities, is sometimes difficult. We have previously shown that trout neutrophil glycogen can be stained by the Thiéry's technique applied to sections of samples processed for the ultrastructural detection of peroxidase or esterase activities, with the simultaneous staining of glycogen and peroxidase, or glycogen and esterase (Afonso et al. 1998a). This double labelling was now used for the comparative study of polysaccharide inclusions in the 2 phagocytes.

Abundant glycogen granules were consistently seen in the cytoplasm of resting or inflammatory neutrophils when adequate techniques were used (Figs. 1, 3A, 4C \& 5). While in peritoneal resting neutrophils, and in blood neutrophils of injected or non-injected fish, the glycogen granules were evenly distributed in the cytoplasmic matrix of most cells (for example, in Fig. 5), in neutrophils from inflamed peritoneal cavities large clusters of glycogen granules were predominant (Fig. 1). The results of the morphometric analysis of 


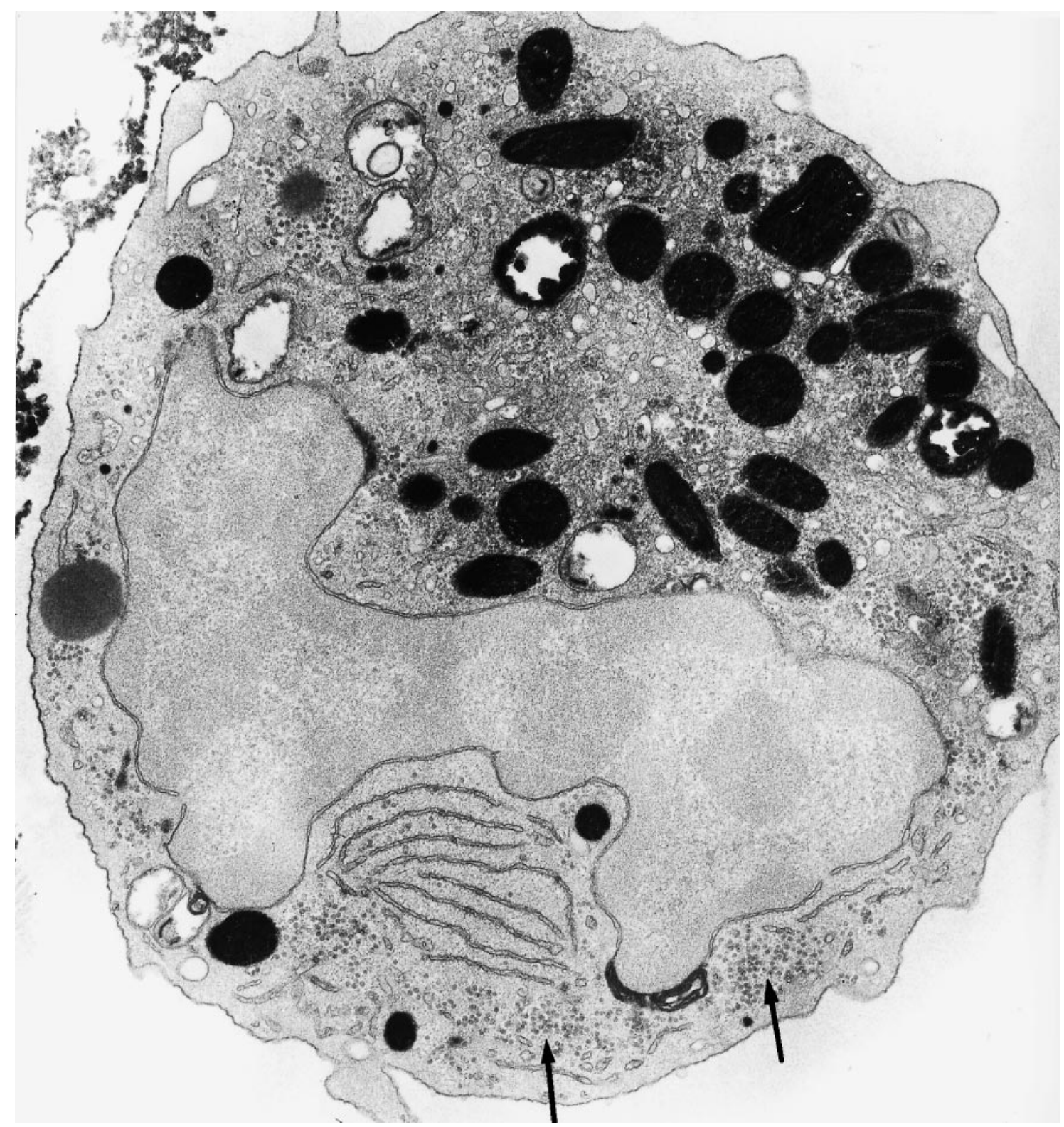

Fig. 1. Oncorhynchus mykiss. Neutrophil in the peritoneal exudate of a rainbow trout injected $24 \mathrm{~h}$ before with $5 \times 10^{7}$ live Yersinia ruckeri. Sample fixed with $0.25 \%$ glutaraldehyde, processed for the detection of peroxidase activity and then fixed with $\mathrm{OsO}_{4}$ supplemented with potassium ferrocyanide; no postfixation with uranyl acetate. Notice the peroxidase-positive granules and the densely contrasted glycogen granules (arrows). Section contrasted with lead citrate. $\times 15000$

glycogen contents in trout neutrophils (Table 1) show that: (1) neutrophils of resting peritoneal cavities had amounts of glycogen granules which were not statistically different from those in blood neutrophils of noninjected trout; (2) glycogen particles were more abundant in neutrophils of the peritoneal cavities injected with IFA or with 2 bacterial inocula as compared to resting peritoneal neutrophils; (3) blood neutrophils of non-injected trout had numbers of glycogen granules which were not statistically different from those in the neutrophils in the blood of trout injected i.p. with the 2 bacterial inocula.

In macrophages, glycogen granules were not seen in resting peritoneal populations and were scarce in inflammatory macrophages (Fig. 4C, Table 1). The significant difference in the glycogen content of the 2 phagocytes explains the strong PAS positivity seen in neutrophils, but not in macrophages by light microscopy of cytospins stained with the Schiff reagent (not shown). 
Table 1. Morphometric assay of amount of glycogen cytoplasmic granules in rainbow trout phagocytes

\begin{tabular}{|c|c|}
\hline Phagocyte & No. of granules $\mu \mathrm{m}^{-2}$ \\
\hline \multicolumn{2}{|l|}{ Non-inflammatory phagocytes } \\
\hline Peripheral blood neutrophils in non-injected trout & $50.80 \pm 13.52$ \\
\hline $\begin{array}{l}\text { Peripheral blood neutrophils in trout injected i.p. wit } \\
\text { killed } Y \text {. ruckeri } 24 \mathrm{~h}+\text { live } Y \text {. ruckeri } 4 \mathrm{~h}\end{array}$ & th $44.45 \pm 12.28$ \\
\hline Resting peritoneal neutrophils & $61.61 \pm 18.27$ \\
\hline Resting peritoneal macrophages & Not detectable \\
\hline \multicolumn{2}{|l|}{ Inflammatory phagocytes } \\
\hline $\begin{array}{l}\text { Peritoneal neutrophils in trout injected i.p. with } \\
\text { Killed Y. ruckeri } 24 \mathrm{~h} \\
\text { Killed } Y \text {. ruckeri } 24 \mathrm{~h}+\text { live } Y \text {. ruckeri } 4 \mathrm{~h} \\
\text { IFA } 44 \mathrm{~h}\end{array}$ & $\begin{aligned} 74.77 & \pm 22.52 \\
121.80 & \pm 42.98 \\
94.57 & \pm 29.98\end{aligned}$ \\
\hline $\begin{array}{l}\text { Peritoneal macrophages in trout injected i.p. with } \\
\text { IFA } 44 \mathrm{~h}\end{array}$ & $14.05 \pm 4.86$ \\
\hline \multicolumn{2}{|c|}{$\begin{array}{l}\text { Comparison (Student's } t \text {-test): Blood neutrophils of non-injected fish/rest- } \\
\text { ing peritoneal neutrophils, } p=0.264 \text {. Blood neutrophils of non-injected } \\
\text { fish/blood neutrophils of i.p. injected fish, } p=0.324 \text {. Resting peritoneal } \\
\text { neutrophils/peritoneal neutrophils Y. ruckeri } 24 \mathrm{~h}, \mathrm{p}=0.339 \text {. Resting peri- } \\
\text { toneal neutrophils/peritoneal neutrophils Y. ruckeri } 24 \mathrm{~h}+Y \text {. ruckeri } 4 \mathrm{~h} \text {, } \\
\mathrm{p}=0.02 \text {. Resting peritoneal neutrophils/peritoneal neutrophils IFA } 44 \mathrm{~h} \text {, } \\
\mathrm{p}=0.049 \text {. Peritoneal macrophages IFA } 44 \mathrm{~h} \text { /peritoneal neutrophils IFA } \\
44 \mathrm{~h}, \mathrm{p}=0.001\end{array}$} \\
\hline
\end{tabular}

\section{Glucose concentration in plasma and inflamed peritoneal exudates}

Glucose concentration in plasma of trout injected with 2 consecutive inocula of Yersinia ruckeri was $187.6 \pm 73.3 \mathrm{mg} 100 \mathrm{ml}^{-1}$. In this situation of peritoneal inflammation, small amounts of ascitic fluid were present. The glucose concentrations were determined in the peritoneal exudates of the same fish, collected after the i.p. injection of $2.0 \mathrm{ml}$ of PBS and then corrected for the volume of the peritoneal fluid. The glucose concentration was $148.0 \pm 42.7 \mathrm{mg} 100 \mathrm{ml}^{-1}$ of the peritoneal fluid. This value is not statistically different from that found in plasma $(\mathrm{p}=0.434)$.

\section{DISCUSSION}

The present results confirm previous data on the influence of fixation methods on the ultrastructural image of glycogen inclusions in prokaryotic and eukaryotic cells (Millonig \& Marinozzi 1968, Vye \& Fischman 1970, Robertson et al. 1975, Robinson et al. 1982, Silva \& Macedo 1983). Double fixation with glutaraldehyde-osmium tetroxide results in strong staining of the granules in bacteria and mammals, whereas in osmium-fixed samples the granules appear electron-transparent (Robertson et al. 1975). The same was now observed for rainbow trout phagocytes. This re- sult has been interpreted on the basis of the strong reaction of glutaraldehyde with glycogen, and of osmium tetroxide with the aldehyde (Glauert 1986). The observation that the polysaccharide granules appear electron-transparent in sections of samples processed for the detection of peroxidase activity can be explained by the use, in the initial glutaraldehyde fixation of the cytochemical protocol, of a low concentration of aldehyde $(0.25 \%)$ to avoid damage to the enzyme activity. However, it was found that an increase in the aldehyde concentration to $1.5 \%$ in the prefixation step of the peroxidase technique resulted in denser images of the polysaccharide inclusions. The enhancement of glycogen contrast described in mammals by the use of osmium tetroxide fixatives containing potassium ferrocyanide (Karnowsky 1971, Robinson et al. 1982) was also found in rainbow trout phagocytes, even when a prefixation with glutaraldehyde was not used (not shown). As reported for other biological materials (Vye \& Fischman 1970, Robertson et al. 1975, Silva \& Macedo 1983), the polysaccharide granules of trout neutrophils were found as electron-transparent areas whenever a postfixation with uranyl acetate was used.

In all situations where the granules appear as electron-transparent areas the Thiéry staining still revealed PAS-positive material. This indicates that the light areas do not result from polysaccharide extraction during the processing for electron microscopy, but rather from a lack of electron contrast.

The use in the present study of a morphometric assay to quantify glycogen in trout phagocytes has the advantage, over the chemical assay, of allowing the analysis of individual leucocytes, whereas the chemical method only gives an averaged value for the total leucocyte population analysed. Moreover, with the use of double-labelling techniques (peroxidase/glycogen and esterase/glycogen) the precise characterization and quantification of glycogen of neutrophils and macrophages was achieved.

Glycogen granules could not be found in resting peritoneal macrophages, and in inflammatory peritoneal macrophages much smaller amounts were found as compared to neutrophils. Also in mammals, macrophages are known to have small reserves of glycogen compared to neutrophils (Carr \& Daems 1980, Parmley 1988). This difference in glycogen content is in accordance with neutrophils being primarily glycolytic cells and macrophages being more capable 


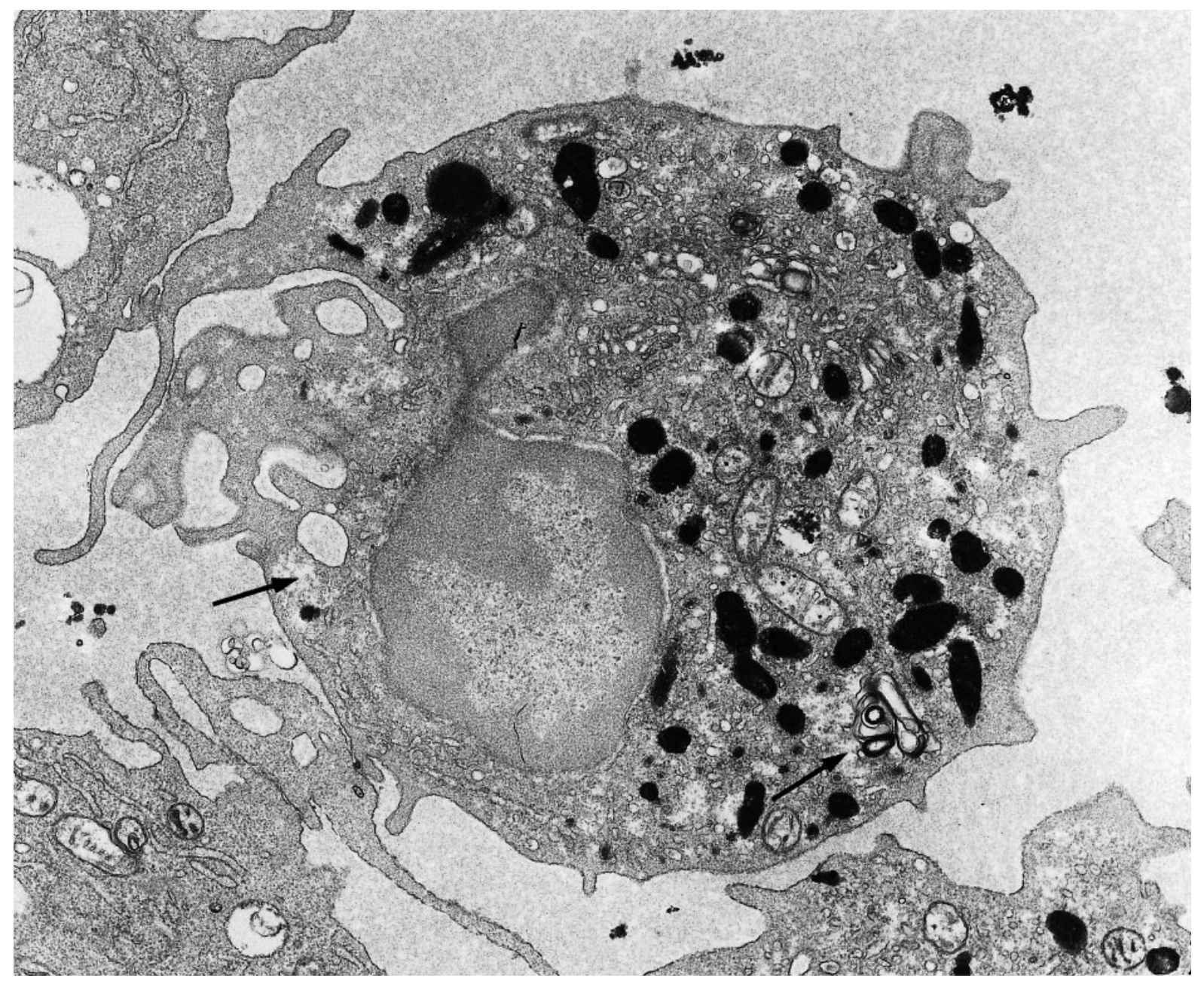

Fig. 2. Oncorhynchus mykiss. Neutrophil in the peritoneal exudate of a rainbow trout injected $24 \mathrm{~h}$ before with $5 \times 10^{7} \mathrm{killed}$ Yersinia ruckeri. Sample fixed with $1.5 \%$ glutaraldehyde, processed for the detection of peroxidase activity and fixed with $\mathrm{OsO}_{4}$ i no postfixation with uranyl acetate. Notice the peroxidase-positive granules and several electron-transparent areas in the cytoplasm (arrows) representing clusters of lightly contrasted glycogen granules. Section contrasted with lead citrate. $\times 15000$

of producing ATP via oxidative phosphorylation (Kilpatrick et al. 1990).

No significant differences in glycogen content were found between blood and resting peritoneal neutrophils. This result, in accordance with the observation that peroxidase content in these 2 neutrophil populations was also similar (Afonso et al. 1999), may be taken as an indication that the small population of neutrophils found in resting peritoneal cavities of rainbow trout is not activated.

As compared to resting peritoneal neutrophils, the glycogen content was found to be slightly higher in neutrophils in the inflamed peritoneal cavity $24 \mathrm{~h}$ after the i.p. injection of killed Yersinia ruckeri and significantly higher following a second injection of live bacteria. A significantly higher glycogen content was also found in peritoneal neutrophils $44 \mathrm{~h}$ after the i.p. injec- tion of IFA as compared to resting peritoneal neutrophils. Afonso et al. (1998a) reported that following the i.p. injection of $5 \times 10^{7}$ Y. ruckeri in rainbow trout, the neutrophils attracted to the peritoneal cavity by the injection of the bacteria were not observed to phagocytose the injected microrganisms. This is so because the resting peritoneal cavity of trout contains abundant resident macrophages but very low numbers of neutrophils (Afonso et al. 1997, 1998a), these numbers increasing significantly several hours after the i.p. inoculation of bacteria (Afonso et al. 1998a). Consequently, the injected bacteria are phagocytosed by the resident macrophages before the arrival of the attracted neutrophils (Afonso et al. 1998a). However, when the bacteria are injected into peritoneal cavities already containing high numbers of neutrophils (by the previous injection of inflammatory agents) neutrophils phago- 

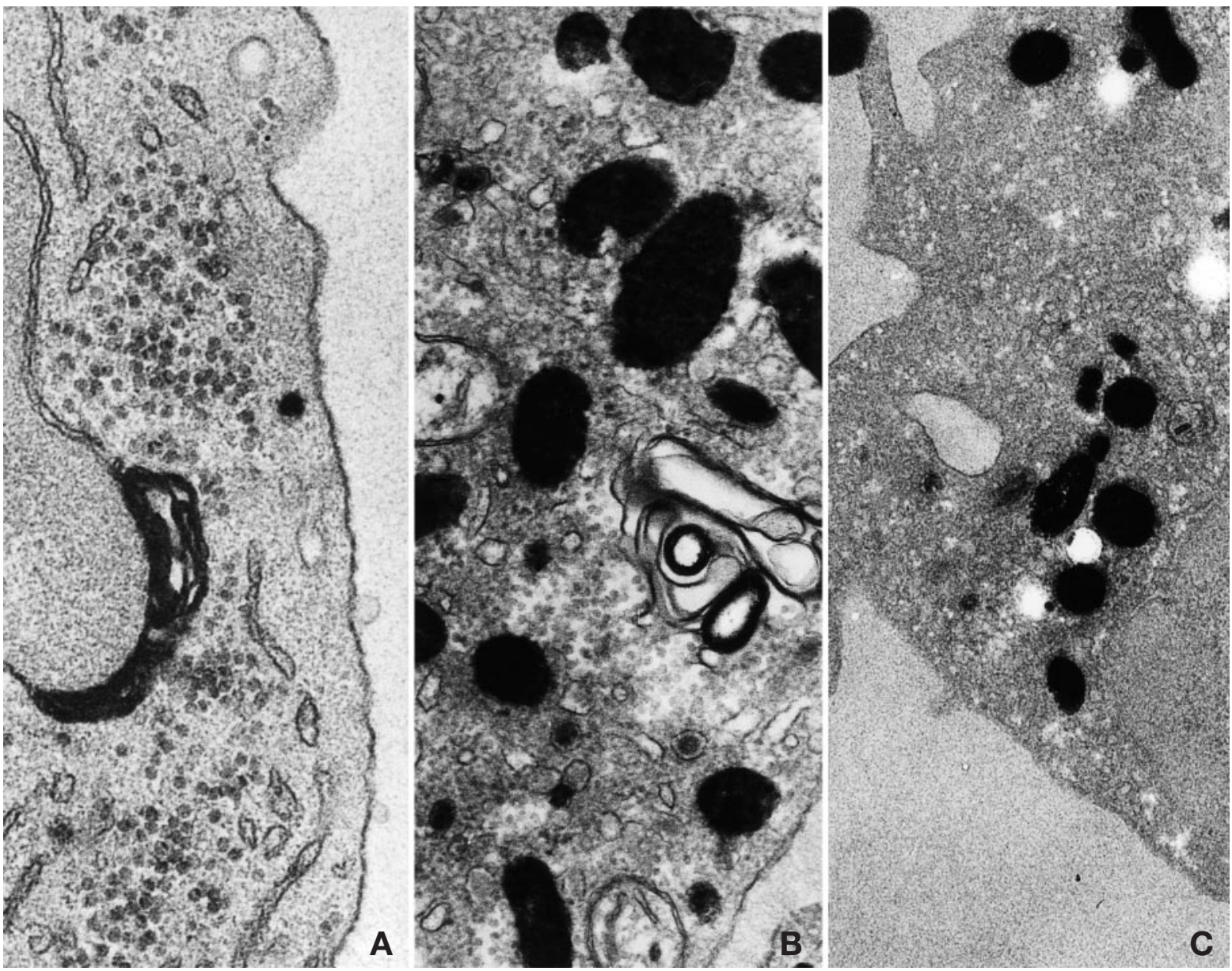

Fig. 3. Oncorhynchus mykiss. (A) Area indicated by the arrows in Fig. 1, showing clusters of densely contrasted glycogen granules (arrows). $\times 49500$. (B) Area indicated by the arrow on the right-hand side of Fig. 2, showing clusters of lightly contrasted glycogen granules. Section contrasted with lead citrate. $\times 46000$. (C) Neutrophil in a resting peritoneal cavity, fixed with $0.25 \%$ glutaraldehyde, processed for peroxidase and fixed with $\mathrm{OsO}_{4}$ i no postfixation with uranyl acetate. Notice the peroxidasepositive granules and several electron-transparent holes in the cytoplasm representing unstained glycogen granules. Section contrasted with lead citrate. $\times 24500$

cytose the microrganisms at a high rate (Afonso et al. 1998a). On the other hand, we found that the mineral oil of the IFA is taken up by the macrophages and by the incoming neutrophils because, for the amount of IFA injected, not all the oil could be taken by the resident macrophages (Afonso et al. 1998a). It seems, therefore, that the neutrophils attracted to the peritoneal cavities when the bacteria are cleared by the macrophages would be engaged in a sort of nonphagocytic activity requiring lower amounts of reserve polysaccharide as compared with situations where the leucocyte is phagocytosing. It has been suggested that glycogen deposits in neutrophils are used up in the respiratory burst that accompanies phagocytosis of microrganisms (Scott 1968, Wilkinson 1977, Weisdorf et al. 1982) and the higher glycogen content of neu- trophils engaged in phagocytosis would be useful. The present observation that the amount of glycogen is not decreased in neutrophils with ingested bacteria is, however, in accordance with the results showing that neutrophil glycogen content only decreases when phagocytosis is carried out in the absence of glucose (Lane \& Lamkin 1985), but not when glucose is available (Stossel et al. 1970). In fact, levels of glucose found in peritoneal fluid in the situation where neutrophils were phagocytosing bacteria were comparable to those found in blood plasma.

An increase in the glycogen content in inflammatory as compared to blood neutrophils has been reported for mammals (Scott \& Cooper 1974, Robinson et al. 1982) and the eel (Nagamura \& Wakabayashi 1985). In mammals and fish, circulating blood neutrophils and 

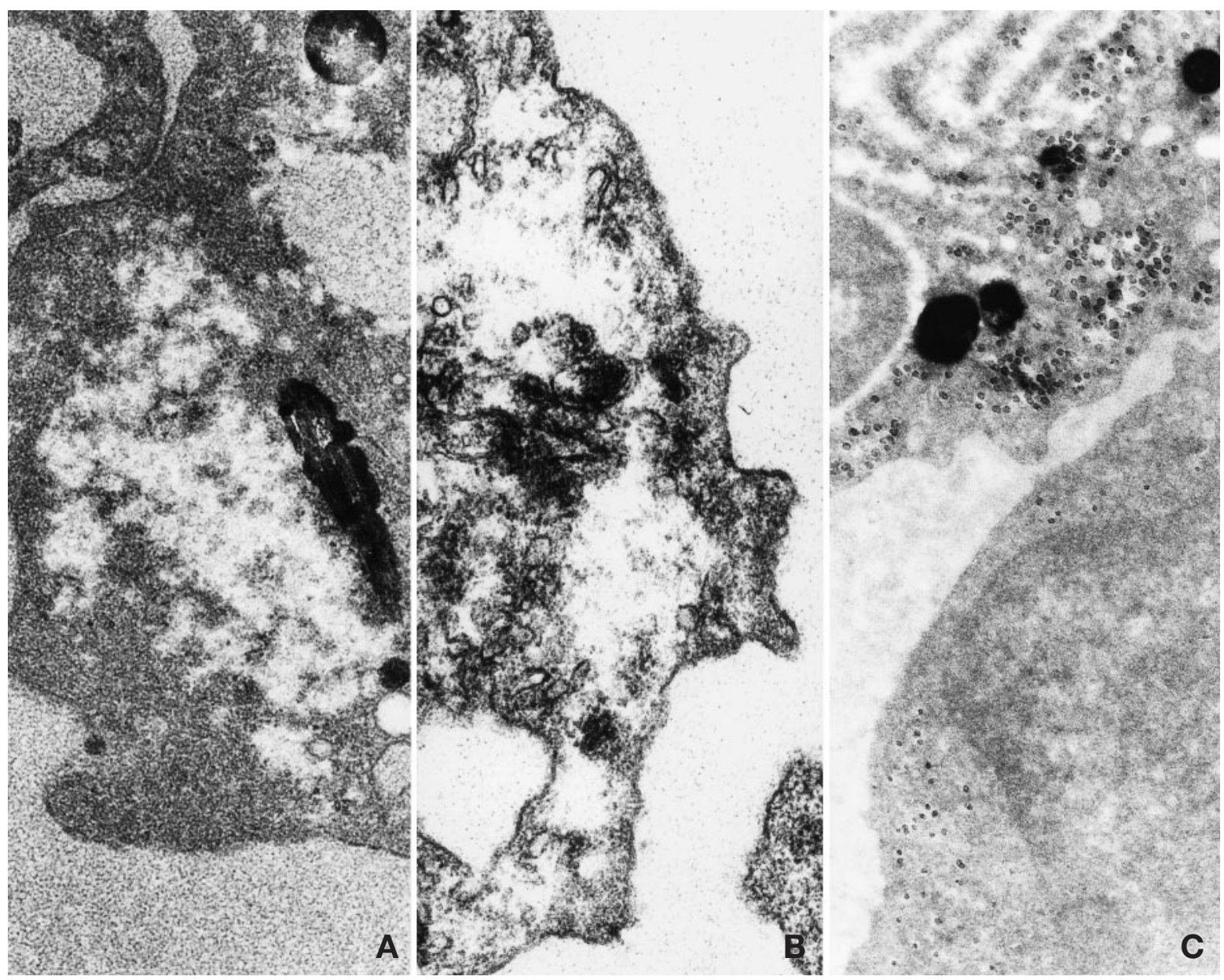

Fig. 4. Oncorhynchus mykiss. (A) Neutrophil in the peritoneal exudate of a rainbow trout injected $24 \mathrm{~h}$ before with $5 \times 10^{7} \mathrm{killed}$ Yersinia ruckeri. Sample fixed with $0.25 \%$ glutaraldehyde, processed for the detection of peroxidase activity and fixed with $\mathrm{OsO}_{4}$; no postfixation with uranyl acetate. Notice the peroxidase-positive granules and electron-transparent areas in the cytoplasm representing unstained clusters of glycogen granules. Section contrasted with uranyl acetate followed by lead citrate. $\times 33600$. (B) Neutrophil in the peritoneal exudate of a rainbow trout injected $44 \mathrm{~h}$ before with Incomplete Freund's Adjuvant (IFA). Sample prefixed with $1.25 \%$ glutaraldehyde $+4 \%$ formaldehyde, fixed with $\mathrm{OsO}_{4}$ and postfixed with uranyl acetate. Clusters of glycogen granules appear as electron-transparent areas. Section contrasted with uranyl acetate followed by lead citrate. $\times 40000$. (C) Same sample as in Fig. 4B with fixation with $0.25 \%$ glutaraldehyde, processed for the detection of peroxidase activity and fixed with $\mathrm{OsO}_{4}$, with no postfixation with uranyl acetate. Section stained by the Thiéry's method. The figure shows portions of a neutrophil (top; with peroxidase-positive granules) and of a macrophage. Notice the clusters of abundant PAS-positive granules in the neutrophil. A few, PAS-positive granules are present in the macrophage; these granules are smaller than those in the neutrophil. $\times 23500$

mature neutrophils of the haematopoietic organ pools are resting cells available to migrate to foci of inflammation (Suzuki \& Iida 1992, Densen et al. 1995). In guinea pigs with the peritoneal cavity rendered inflamed by the i.p. injection of casein or LPS (lipopolysaccharide), Scott \& Cooper (1974) found that at $24 \mathrm{~h}$ post-injection, the amount of glycogen of peritoneal neutrophils was increased 7-fold, while, at the same time, blood neutrophils showed only a 1.6-fold increase in glycogen content. This observation is in accordance with those of Wulff (1962) and Robinson et al. (1982), and with our finding that trout with peritoneal cavities inflamed by 2 consecutive i.p. injections of bacteria have blood neutrophils with glycogen granules in amounts similar to those of the non-injected fish. These results suggest that the increase in glycogen content of the inflammatory neutrophils observed in the inflamed peritoneal cavities of mammals (Scott \& Cooper 1974, Robinson et al. 1982) and fish (present results) would occur during the migration from the blood or in the inflammatory foci rather than in the haematopoietic tissues or peripheral blood. However, some reports described an increase in PAS staining of blood neutrophils of the eel (Nagamura \& Wakabayashi 1985, Hine \& Wain 1988) or rainbow trout (Lamas et al. 1994) after the injection of bacteria or bacterial 


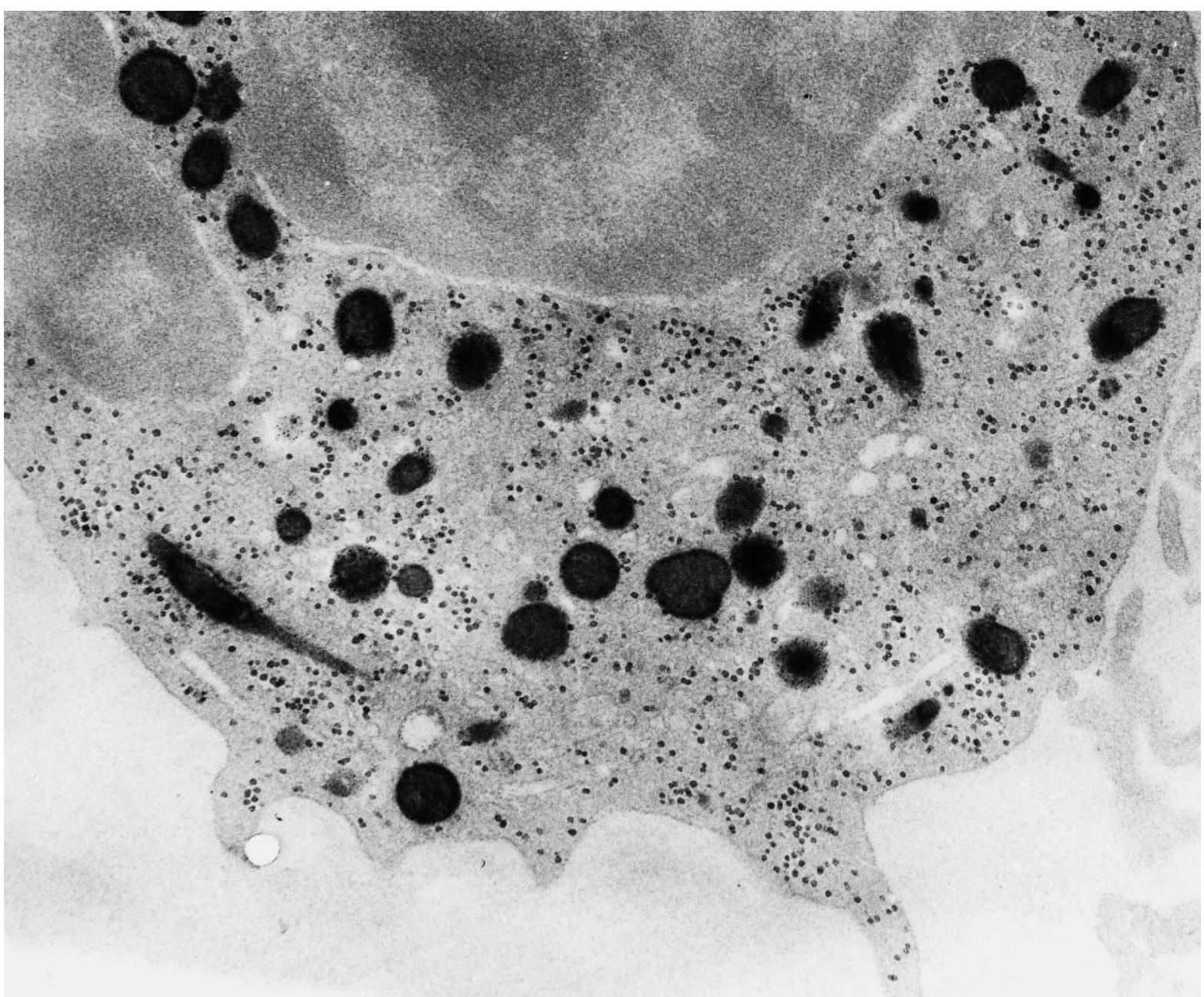

Fig. 5. Same sample and same fixation as in Fig. 3C, but section stained by the Thiéry's technique. Non-clustered PAS-positive glycogen particles and peroxidase-positive granules are uniformly distributed in the cytoplasmic matrix of a neutrophil. $\times 19200$

products. These conflicting results may well be explained by the use in the above studies of high doses of inflammatory agents which produced progressive disease and are likely to induce a systemic inflammatory reaction, therefore affecting circulating neutrophils. In contrast, in our study, transient inflammatory situations restricted to the peritoneal cavity were used.

The polysaccharide granules we found in inflammatory macrophages were of 2 types. One had a diameter smaller than that of neutrophil granules. The other seems to represent neutrophil glycogen transferred to macrophages. This would occur through the same mechanisms described for the transfer of other neutrophil components, like peroxidase (Afonso et al. 1998b). It is tempting to consider that the glycogen particles transferred from neutrophils to macrophages would be used by the macrophages for their own metabolic activities, as was suggested by Rebuck et al. (1968).
Acknowledgements. The authors are grateful to Prof. Alberto Villena and Dr Jorge Pedrosa for helpful discussions and to Prof. António C. Castro for supplying the fish used in the present work. We thank Prof. A. Freitas da Fonseca for the glucose assays and Prof. J. P. Cabral for the help with statistics. The excellent technical assistance provided by Miss Irene Barros and Mr Adão Silva is gratefully acknowledged.

\section{LITERATURE CITED}

Afonso A, Ellis AE, Silva MT (1997) The leucocyte population of the unstimulated peritoneal cavity of rainbow trout (Oncorhynchus mykiss). Fish Shellfish Immunol 7:335-348

Afonso A, Lousada S, Silva J, Ellis AE, Silva MT (1998a) Neutrophil and macrophage responses to inflammation in the peritoneal cavity of rainbow trout Oncorhynchus mykiss. A light and electron microscopic cytochemical study. Dis Aquat Org 34:27-37

Afonso A, Silva J, Lousada S, Ellis AE, Silva MT (1998b) Uptake of neutrophils and neutrophilic components by macrophages in the inflamed peritoneal cavity of rainbow trout (Oncorhynchus mykiss). Fish Shellfish Immunol 8:319-338 
Afonso A, Oliveira C, Ellis AE, Silva MT (1999) Peroxidase activity as a measure of neutrophil populations in inflammatory peritoneal exudates of rainbow trout, Oncorhynchus mykiss (Walbaum). J Fish Dis 22:133-142

Bozdech MJ, Bainton DE (1981) Identification of alpha naphtyl butyrate esterase as a plasma membrane ectoenzyme of monocytes and as a discrete intracellular membrane-bounded organelle in lymphocytes. J Exp Med 153:182-195

Carr I, Daems WT (1980) The macrophage. A bird's-eye view. In: Carr I, Daems WT (eds) The reticuloendothelial system. A comprehensive treatise, Vol 1. Plenum Press, New York, p 1-18

Densen P, Clark RA, Nauseef WM (1995) Granulocytic phagocytes. In: Maudell GL, Bennett JE, Dolin R (eds) Principles and practice of infection diseases, 4th edn. Churchill Livingstone, Edinburgh, p 78-102

Drochmans P (1962) Morphologie du glycogène. Étude au microscope électronique de colorations négatives du glycogène particulaire. J Ultrastruct Res 6:141-155

Ellis AE (1977) The leucocytes of fish: a review. J Fish Biol 11:453-491

Glauert AM (1986) Fixation, dehydration and embedding of biological specimens. In: Glauert AM (ed) Practical methods in electron microscopy, Vol 3, Part I. North-Holland Publ Co, Amsterdam, p 31-38

Hine PM, Wain JM (1988) Characterization of inflammatory neutrophils induced by bacterial endotoxin in the blood eels, Anguilla australis. J Fish Biol 32:579-592

Karnowsky MJ (1971) Use of Ferrocyanide-reduced osmium tetroxide in electron microscopy. In: Proc 14th Ann Meeting Am Soc Cell Biol, p 146

Kilpatrick L, Garty BZ, Lundquist KF, Hunter K, Stanley CA, Baker L, Douglas SD, Korchak HM (1990) Impaired metabolic function and signalling defects in phagocytic cells in glycogen storage disease type $1 \mathrm{~b}$. J Clin Invest 86 : 196-202

Lamas J, Santos Y, Bruno DW, Toranzo AE, Anadón R (1994) Non-specific cellular responses of rainbow trout to Vibrio anguillarum and its extracellular products (ECPs). J Fish Biol 45:839-854

Lane TA, Lamkin GE (1985) Glycogen metabolism in stored granulocytes. Transfusion 25:246-250

MacArthur JI, Fletcher TC (1985) Phagocytosis in fish. In: Manning MJ, Tatner MF (eds) Fish immunology. Academic Press, New York, p 29-46

Millonig G, Marinozzi V (1968) Fixation and embedding in electron microscopy. In: Barer R, Cosslett VE (eds) Advances in optical and electron microscopy, Vol 2. Academic Press, New York, p 251-341

Nagamura Y, Wakabayashi H (1985) Changes in glycogen content of neutrophils in eel, Anguilla japonica by bacterial infection. Fish Pathol 20:389-394

Parmley RT (1988) Mammals. In: Rowley AF, Ratcliffe NA (eds) Vertebrate blood cells. Cambridge University Press, Cambridge, p 337-424

Rebuck JW, Whitehouse FW, Noonan SM (1968) Energization of lymphocytes transforming to macrophages in human inflammation. Biochem Pharmacol Suppl, p 159-170

Editorial responsibility: David Bruno, Aberdeen, Scotland, UK
Robbins D, Fahimi HD, Cotran RA (1971) Fine structural cytochemical localization of peroxidase activity in rat peritoneal cells, mononuclear cells, eosinophils and mast cells. J Histochem Cytochem 19:517-575

Robertson JG, Lyttleton P, Williamson KI, Batt RD (1975) The effect of fixation procedures on the electron density of polysaccharide granules in Nocardia corallina. J Ultrastruct Res 50:321-332

Robinson JM, Karnovsky ML, Karnovsky MJ (1982) Glycogen accumulation in polymorphonuclear leucocytes, and other intracellular alterations that occur during inflammation. J Cell Biol 95:933-942

Rowley AF, Hunt TC, Page M, Mainwaring G (1988) Fish. In: Rowley AF, Ratcliffe NA (eds) Vertebrate blood cells. Cambridge University Press, Cambridge, p 19-128

Scott RB (1968) The role of glycogen in blood cells. New Engl J Med 278:1436-1444

Scott RB, Cooper LB (1974) Leucocyte glycogen response in inflammatory exudates. Br J Haematol 26:485-496

Silva MT, Macedo PM (1983) The interpretation of the ultrastructure of mycobacterial cells in transmission electron microscopy of ultrathin sections. Int J Leprosy 51:225-234

Silva MT, Macedo PM (1987) Improved Thiéry staining for the ultrastructural detection of polysaccharides. J Submicrosc Cytol 19:677-681

Silva MT, Santos-Mota JM, Melo JVC, Carvalho-Guerra F (1971) Uranyl salts as fixatives for electron microscopy. Study of the membrane ultrastructure and phospholipid loss in bacilli. Biochim Biophys Acta 233:513-520

Silva MT, Appelberg R, Silva MNT, Macedo PM (1987) In vivo killing and degradation of Mycobacterium aurum within mouse peritoneal macrophages. Infect Immun 55: 2006-2016

Stossel TP, Murad F, Mason RJ, Vaughan M (1970) Regulation of glycogen metabolism in polymorphonuclear leucocytes. J Biol Chem 245:6228-6234

Suzuki Y, Iida T (1992) Fish granulocytes in the process of inflammation. Annu Rev Fish Dis 1:140-160

Thiéry JP (1967) Mise en évidence des polysaccharides sur coupes fines en microscopie électronique. J Microsc 6: 987-1018

Thuvander A, Norrgren L, Fossum C (1987) Phagocytic cells in blood from rainbow trout, Samo gairdneri (Richardson), characterized by flow cytometry and electron microscopy. J Fish Biol 31:197-208

Vye MV, Fischman DA (1970) The morphological alteration of particulate glycogen by en bloc staining with uranyl acetate. J Ultrastruct Res 33:278-291

Weisdorf DJ, Craddock PR, Jacob HS (1982) Glycogenolysis versus glucose transport in human granulocytes: differential activation in phagocytosis and chemotaxis. Blood 60: 888-893

Wilkinson PC (1977) Non-specific cells: Granulocytes, mononuclear phagocytes and mast cells. In: Holborow EJ, Reeves WG (eds) Immunology in medicine. Academic Press, London, p 231-264

Wulff HR (1962) Histochemical studies of leucocytes from an inflammatory exudate. Glycogen and phosphorilase. Acta Haematol 28:86-93

Submitted: December 22, 1999; Accepted: May 22, 2000 Proofs received from author(s): July 19, 2000 